\title{
Ge-conservación (España)
}

\author{
Rocío Bruquetas Galán
}

a revista Ge-conservación, un proyecto que el Grupo Español del International Institute for Conservation of Historic and Artistic Works (GEIIC) acariciaba desde el 2001, publicó su primer número en diciembre del 2008 (Figura 1). De origen diseñada como una publicación digital, de acceso abierto y regida por el modelo de revisión anónima por pares, aspiraba a ser un vehículo de difusión de las investigaciones y propuestas metodológicas que se realizan en el ámbito de la conservación y restauración del patrimonio cultural de España. La necesidad de un medio de tales características se justificaba, entre otros hechos, por la escasez tanto de difusión de trabajos sobre el tema como de publicaciones periódicas profesionales de este tipo en idioma español. Mientras que las existentes hasta el momento estaban vinculadas con instituciones públicas o académicas, en este caso la propuesta editorial partía de una asociación, cuyo propósito era dotar de voz al sector en general, tanto público como privado, y dar a conocer sus investigaciones, actuaciones y debates críticos mediante un foro común y ágil, accesible a todos los profesionales.

Si bien desde 1996, año de su fundación como grupo regional del IIC que tiene su sede en Londres, el GEIIC, esta asociación sin ánimo de lucro, ya venía cumpliendo un importante papel de cohesión profesional, al servir de enlace y comunicación entre los ámbitos particular, institucional y académico, así como con colectivos de otros países, y tenía en el campo de las publicaciones una de sus principales aspiraciones. En la actualidad en el grupo contamos con un total de trescientos quince socios, cinco de ellos, empresariales, y doce, institucionales. Desde el principio, nuestra sede ha sido el Instituto del Patrimonio Cultural de España (IPCE), perteneciente al ahora Ministerio de Educación, Cultura y Deporte, MECD (Madrid, España), con excepción de algunos años, cuando cambiamos al Museo Nacional Centro de Arte Reina Sofía (MNCARS, Madrid, España).

En "reconocimiento a su amplia trayectoria como asociación y exponente de la participación de la sociedad civil en la conservación, investigación, formación y difusión del patrimonio cultural" (MECD 2014), en el 2011 GEIIC recibió el Premio Nacional de Conservación y Restauración de Bienes Culturales del MECD — primera vez que éste lo otorgó a una organización de carácter asociativo y profesional, en este caso, con una amplia proyección en la comunidad de conservadores y restauradores-, lo

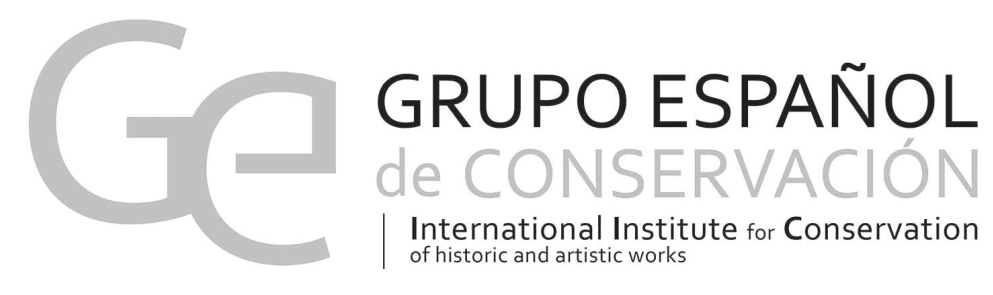

FIGURA 1. Logotipo de Ge-conservación (Cortesía: Ge-conservación, GEIIC). 
que significó una distinción a todo un colectivo interesado en involucrarse en la preservación de su patrimonio cultural.

Hoy Ge-conservación, tras cinco años de publicación, sin duda ha cumplido satisfactoriamente los objetivos del GEIIC, entre los cuales está promover la educación y la investigación en todas las ramas de la ciencia y la práctica de la conservación del patrimonio cultural, así como la aplicación y difusión de sus conocimientos, metodología y criterios; potenciar el intercambio de la información técnica y profesional entre sus miembros; editar publicaciones, realizar exposiciones y organizar congresos, conferencias y reuniones en el campo de la conservación del patrimonio histórico y artístico y, finalmente, cooperar con todos aquellos profesionales de la conservación - tanto instituciones como empresas y particulares- que lo soliciten (GEIIC 2014a). Cabe señalar que la oferta de cursos, especializados o divulgativos, que en un inicio constituían el principal eje de actividad de la asociación, se amplió conforme ésta crecía, junto con sus posibilidades de colaborar con otras instituciones. Así, entre los organismos públicos que han contribuido y apoyado en sucesivas ocasiones al GEIIC se encuentran el propio IPCE-MECD y el Instituto Valenciano de Conservación y Restauración (IVCR); universidades como la Complutense de Madrid (UCM), la de Alcalá de Henares (UAH) en Madrid, y las de Valencia (UV) y Barcelona (UB); museos como el Cerralbo, el del Traje, el MNCARS, el del Prado (los cuatro, en Madrid), el Nacional de Arte de Catalunya (MNAC), el de Bellas Artes de Valencia (MBAV); organismos de investigación como el Instituto Tecnológico Textil (Aitex) de Alcoy, Valencia, y el Instituto de Química Física Rocasolano (IQFR), del Consejo Superior de Investigaciones Científicas (CSIC), España, así como numerosas organizaciones privadas, asociaciones y fundaciones de este mismo país. Entre estas últimas, la Fundación Duques de Soria (FDS, España) -institución cultural sin ánimo de lucro con sede en Soria, creada en 1989 por iniciativa y bajo la presidencia de honor de la Infanta doña Margarita de Borbón y de don Carlos Zurita, duques de Soria-, ha sido fundamental: entre otras actividades conjuntas, patrocinó y coeditó el número 0 de Ge-conservación (FDS 2009).

Ahora bien, en el espíritu de promover el intercambio de conocimientos entre profesionales que, como se dijo arriba, ha sido otro de los fines básicos del GEIIC, se han organizado congresos internacionales cada tres años, todos con sedes en España. El primero, Conservación del patrimonio: evolución y nuevas perspectivas (GEIIC 2014b), se celebró en Valencia en el 2002 en torno de comunicaciones y mesas de debate sobre tres temas esenciales: formación y ejercicio profesional; metodología, criterios y técnicas de intervención e investigación, y nuevas tecnologías. De gran interés resultó el segundo, internacional, verificado en el paraninfo de la UB del 9 al 11 de noviembre del 2005, titulado Investigación en conservación y restauración del patrimonio cultural, con la colaboración del MNAC (GEIIC 2014c). Este congreso, más especializado, congregó a alrededor de 400 profesionales que presentaron y escucharon trabajos sobre criterios y planteamientos metodológicos aplicados a las diversas áreas de la conservación, el empleo de las nuevas tecnologías en los estudios y técnicas de intervención, y la investigación de fuentes documentales. Le siguieron: el de Oviedo, en noviembre del 2007, La Conservación infalible: de la teoría a la realidad, con el apoyo del Principado de Asturias (GEIIC 2014d); el de Cáceres, La restauración del siglo XXI: función, estética e imagen, en noviembre del 2009 (GEIIC 2014e), y el más reciente, Criterios de calidad en intervenciones, realizado en la UCM en abril del 2012 (GEIIC 2014f).

El gran interés mostrado en nuestras actividades no hacía más que evidenciar la necesidad que tenemos los profesionales de la conservación de intercambiar información. Para todas ellas, como para muchas otras iniciativas, la página web (GEIIC 2014a) ha supuesto una dinámica herramienta de comunicación: mediante ésta se difunden las actividades no sólo del grupo sino también las de carácter nacional e internacional relacionadas con el campo de estudio, al punto de convertirse en un lugar de referencia para el mundo hispanohablante. La web, además de agilizar las tareas de organización y difusión de la asociación y sus proyectos, nos ha permitido llegar a otros países europeos y americanos, así como conocer sus trabajos e inquietudes.

Entre las iniciativas que han aportado una mayor vitalidad a la asociación están los diferentes grupos de trabajo, formados gradualmente a lo largo de estos años. El veterano es el de Arte Contemporáneo: comenzó sus actividades en el 2002 y en los meses de febrero de cada año, en coincidencia con la Feria Internacional de Arte Contemporáneo, Arco, que se lleva a cabo en la Institución Ferial de Madrid (Ifema), realiza sus reuniones en el MNCARS; las actas de éstas, que se publican anualmente, hoy se evalúan como un referente de calidad para la información sobre nuevas tendencias en conservación del arte actual (GEIIC 2014g). El Grupo de Retablos se creó con base en la enorme aceptación y poder de convocatoria que alcanzaron los cursos monográficos celebrados desde el 2002 sobre este tema en Madrid (UCM) y Valencia (UV) y Museo de Bellas Artes de Valencia San Pío V (MBAV), que con posterioridad se publicaron impresas y digitalmente (GEIIC 2014a). El Grupo de Conservación Preventiva (GEIIC 2014a), por su parte, ha sido el promotor de una de las primeras y más interesantes colaboraciones internacionales del GEIIC, concretamente con México y Brasil, países con los que organizó una serie de reuniones técnicas para la elaboración de un documento sobre buenas prácticas en el tratamiento de los bienes culturales en exposiciones temporales: Conservación preventiva y procedimientos en exposiciones temporales, editado en español y portugués (Fernández 2008). Los grupos Científico-Técnico, de Piedra, Artes Decorativas, Foto- 
grafía y Patrimonio Industrial, incorporados más tarde, han desarrollado tanto su propio espacio, en la web, con información sobre tareas específicas y líneas de trabajo, documentación e investigación, como diversas actividades formativas y divulgativas - cursos, jornadas profesionales y publicaciones (GEIIC 2014a)—, cuyo interés nos impulsó a recoger sus contenidos e iniciar una línea de publicaciones en papel, en formato electrónico y, más tarde, en acceso abierto en la red por medio de la página web del grupo (GEIIC 2014a).

Los ricos debates suscitados en el mencionado congreso de Barcelona (GEIIC 2014c) en relación con la investigación en conservación del patrimonio cultural fueron claves para la puesta en marcha del proyecto de la revista digital: la elevada participación de público puso de relieve, además del interés y el alto grado de preparación de nuestra profesión para abordar su vertiente científica, el deseo generalizado por sacarla de los talleres aislados, ámbito todavía habitual para la práctica de nuestro trabajo. Se hizo patente, entonces, la necesidad de crear una revista en la que poder dar a conocer los avances de la investigación y las puestas al día de técnicas y metodologías de trabajo, que contribuyera al desarrollo científico y a la difusión e intercambio de conocimientos en materia de conservación-restauración. El resultado fue Ge-conservación (Figura 2).

Uno de los propósitos inmediatos de esta publicación era desarrollar los vínculos culturales entre las comunidades autónomas de España y extender la comunicación con aquellos países que comparten las lenguas española y portuguesa y, por lo tanto, hacerla llegar a todo el ámbito iberoamericano. El patrocinio de la Fundación Duques de Soria (FDS 2009) para la coedición del número cero fue vital para consolidar la proyección hacia Portugal e Iberoamérica. Así, la zona de distribución de la revista - que ya contaba con colaboraciones iberoamericanas - se ha ampliado a países de idioma portugués (incluido Brasil y algunos de África).

La revista Ge-conservación (GEIIC 2014h) está especializada en la publicación de contenidos que comuniquen resultados de investigaciones académicas originales en cualquier área de conocimiento relacionada con la conservación del patrimonio cultural y de intervenciones de conservación-restauración de interés, ya sea por el significado cultural del propio bien, por los resultados obtenidos o por la novedad de las propuestas metodológicas, así como de artículos de opinión sobre aspectos teóricos de la conservación y la restauración (Figura 3). Se estructura en tres secciones: la primera comprende contribuciones, mediante un texto o una entrevista, de personalidades destacadas en diferentes áreas, cuya trayectoria constituye un referente profesional. Entre otros muchos, hemos tenido el honor de contar con especialistas de la altura de Gaël de Guichen, Magdalena Krebs, René de la Rie, Paolo Cremonesi, Concha Cirujano, Araceli Gabaldón, Arsenio Sánchez Hernampérez, Beatriz Ramos de Vasconcelos

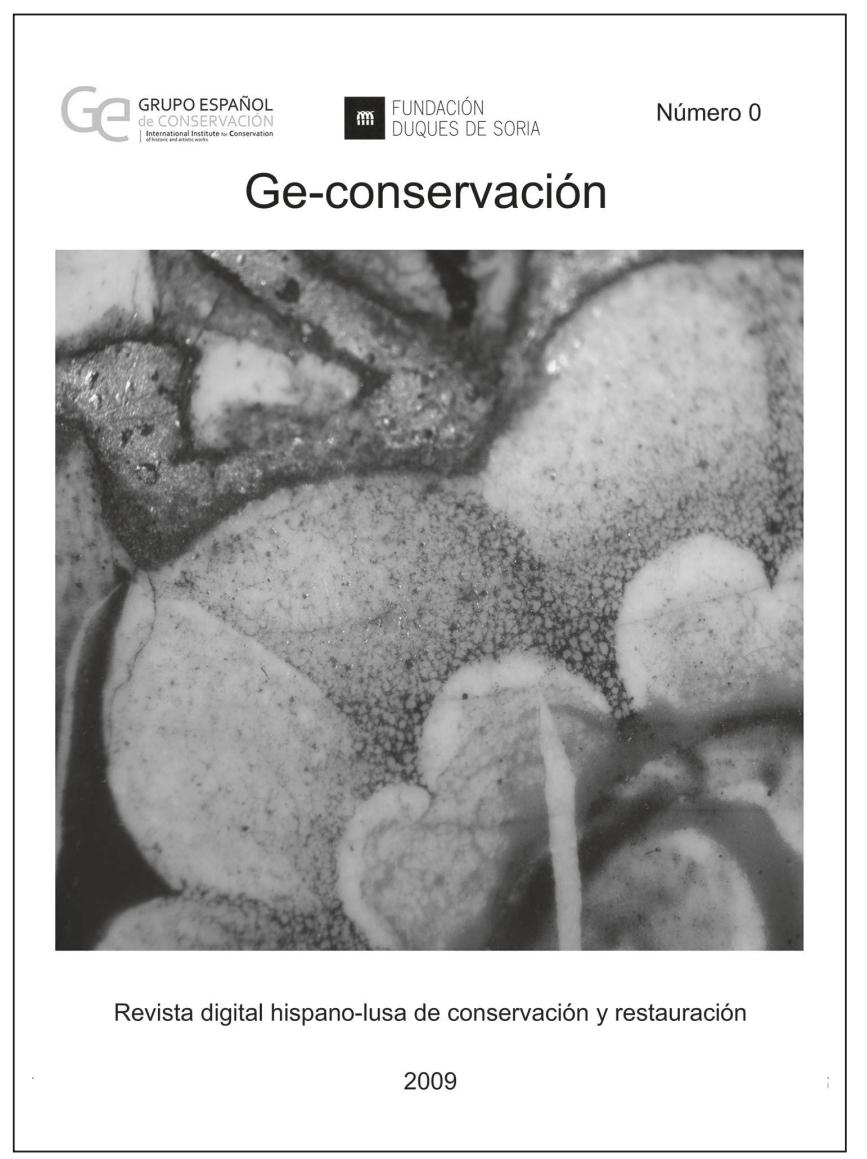

FIGURA 2. Portada del número 0 de Ge-conservación, 2009 (Cortesía: Ge-conservación, GEIIC).

Coelho, Fernando dos Santos Antunes, Alfonso Muñoz Cosme, Edson Motta, Carmen Añón Feliu, Vasco Fassina e Isabel García Fernández. La segunda sección, el cuerpo principal, corresponde a artículos seleccionados en los que prima el espíritu crítico, el carácter multidisciplinar y la fundamentación de los criterios y la metodología seguidos (Figura 4), y la tercera incluye algunas reseñas de libros sobre las novedades que se publican en nuestro campo, elaboradas por los miembros del consejo de redacción y otros especialistas (GEIIC 2014h).

Quienes evalúan la calidad de los artículos que se someten a publicación son pares que conforman un amplio comité científico de conocedores de diversas áreas de la conservación-restauración y profesionales y académicos de diferentes ámbitos que trabajan de forma anónima. El equipo de gestión está formado por la dirección, compartida en estos momentos por Ana Calvo Manuel (Facultad de Bellas Artes, FBA-UCM, España) y Rocío Bruquetas Galán (IPCE-MECD), y un consejo de redacción compuesto por ocho miembros que deciden la orientación general de la revista y los contenidos de cada número: Marisa Gómez (IPCE-MECD), Emilio Ruiz de Arcaute Martínez (Diputación Foral de Álava, España), Margarita San Andrés (FBA-UCM), Emma García Alonso (IPCE-MECD), Ana Laborde 


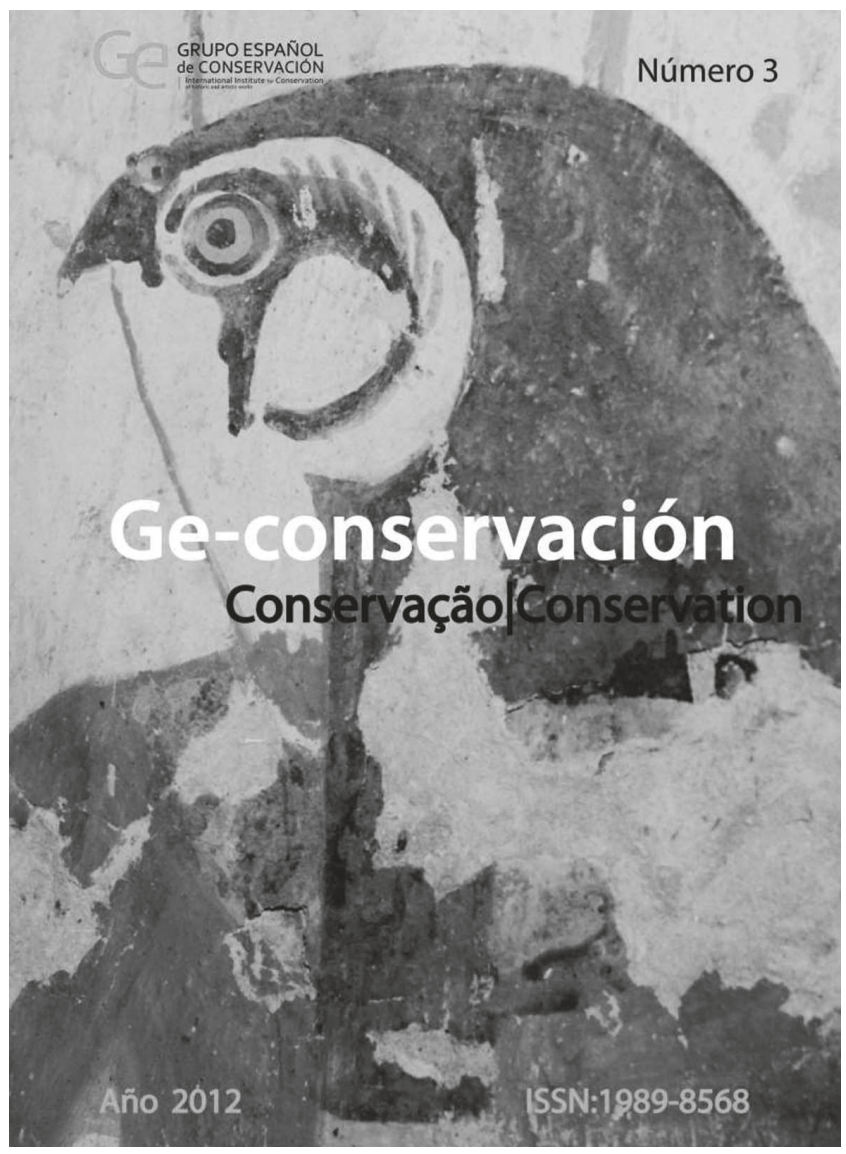

FIGURA 3. Portada del número 3 de Ge-conservación, 2012 (Cortesía: Ge-conservación, GEIIC).

(IPCE-MECD), María Aguiar (Universidad Católica Portuguesa, UCP, Oporto, Portugal), Emilio Cano (Centro Nacional de Investigaciones Metalúrgicas, Cenim del CSIC, España) y Sandra Zetina Ocaña (Universidad Nacional Autónoma de México, UNAM, México).

La secretaría de edición, ocupada, aun hasta diciembre del 2013, por Christhiam Fiorentino y, desde esa fecha, por Concepción de Frutos (ambos, restauradores independientes), como encargada del sistema de gestión de la revista progresivamente ha ido ganando en eficiencia, al adoptar el programa de administración y edición electrónica de revistas científicas Open Journal System (OJS), con el que se ha conseguido, hacia el exterior, ampliar su difusión y visibilidad en el medio académico, científico y profesional y mejorar su indexación y, hacia dentro, una mayor transparencia en las políticas de la publicación. En este momento figura ya indexada en las bases de datos bibliográficas de conservación más importantes: The Bibliographic Database of the Conservation Information Network (BCIN 2014) y Abstracts of International Conservation Literature, AATA Online (GCI 2014), del Getty Conservation Institute (GCI), así como en otros repertorios de publicaciones periódicas científicas y de humanidades de gran peso: ISOC del CSIC (CSIC 2014 ), Scopus (Elsevier 2014) y Dialnet (Unirioja 2014).

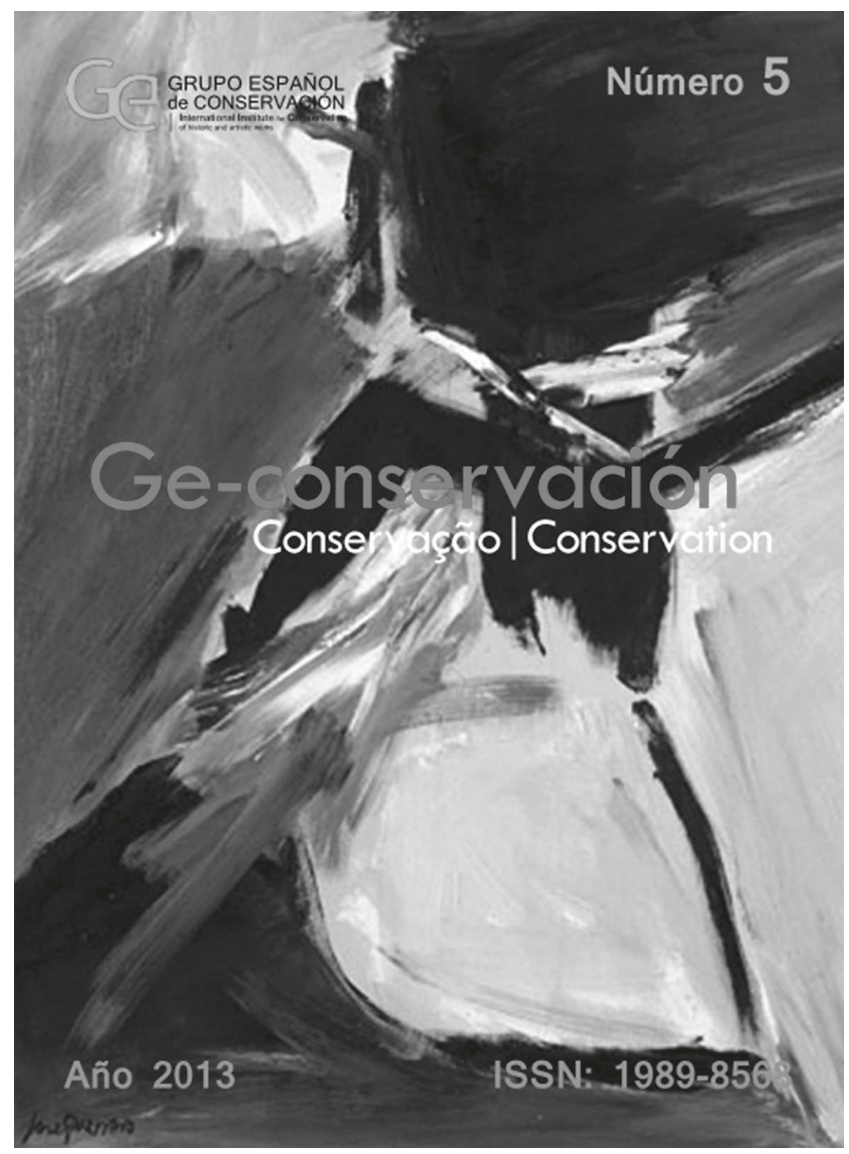

FIGURA 4. Portada del número 5 de Ge-conservación, 2013 (Cortesía: Ge-conservación, GEIIC).

Por último, Ge-conservación, que es una muestra más del interés del GEIIC por involucrar a profesionales latinoamericanos en las actividades que viene desarrollando, ha ampliado no sólo el marco geográfico de su difusión sino también la demanda de profesionales e investigadores que solicitan publicar en ella, lo cual nos satisface enormemente. Un factor favorable en este sentido es el idioma compartido y los permanentes lazos de intercambio entre nuestras sociedades y culturas, lo que hace deseable una comunicación fluida que nos enriquezca mutuamente en el conocimiento de nuestro patrimonio cultural y su conservación.

\section{Referencias}

\section{BCIN}

2014 The Bibliographic Database of the Conservation Information Network (BCIN), documento electrónico [página web] disponible en [http://www.bcin.ca], consultada en julio del 2014. CSIC

2014 ISOC, base de datos de ciencias sociales y humanidades del CSIC, documento electrónico [página web] disponible en [http://bddoc.csic.es:8080], consultada en julio del 2014. 
Elsevier (editoral)

2014 Scopus, base de datos de la editorial Elsevier, documento electrónico [página web] disponible en [http://www.elsevier.com/online-tools/scopus], consultada en julio del 2014. FDS

2009 Fundación Duques de Soria, documento electrónico [página web] disponible en [http://www.fds.es], consultada en julio del 2014.

Fernández, Charo (coord.)

2008 Conservación preventiva y procedimientos en exposiciones temporales, Madrid, GEIIC/FDS.

GCI

2014 Abstracts of International Conservation Literature, AATA Online, base de datos del $\mathrm{GCl}$, documento electrónico [página web] disponible en [http://aata.getty.edu], consultado en julio del 2014.

GEIIC

2014a Grupo Español de Conservación, GEIIC, documento electrónico [página web] disponible en [http://www.ge-iic. com], consultado en julio del 2014.

2014b Actas del I Congreso Internacional del GEIIC. Conservación del patrimonio: evolución y nuevas perspectivas, Valencia, 25 al 27 de noviembre del 2002, documento electrónico disponible en [http://ge-iic.com/index.php?option=com_cont ent\&task=view\&id=32\&ltemid=40], consultado en julio del 2014.

2014c Actas del II Congreso Internacional del GEIIC. Investigación en conservación y restauración del patrimonio cultural, UB, España, 9 al 11 de noviembre del 2005, documento electrónico disponible en [http://ge-iic.com/index. php?option=com_content $\&$ task=view $\& i d=40 \&$ Itemid $=40$ ], consultado en julio del 2014. 2014d Actas del III Congreso Internacional del GEIIC. La conservación infalible: de la teoría a la realidad, Oviedo, 21 al 23 de noviembre del 2007, documento electrónico disponible en [http://ge-iic.com/index.php?option=com_content\&task=vie w\&id=287\&ltemid=40], consultado en julio del 2014.

2014 e Actas del IV Congreso Internacional del GEIIC. La restauración del siglo XXI: función, estética e imágenes, Cáceres, 25 al 27 de noviembre del 2009, documento electrónico disponible en [http://ge-iic.com/index.php?option=com_content\&tas k=view\&id=590\&ltemid=40], consultado en julio del 2014 . $2014 \mathrm{f}$ Actas del V Congreso Internacional del GEIIC. Criterios de calidad en intervenciones, Madrid, 18 al 20 de abril del 2012, documento electrónico disponible en [http://ge-iic. com/index.php?option $=$ com_content \&task=view\&id=867\&lt emid=40], consultado en julio del 2014.

2014g Actas de las jornadas realizadas por el Grupo de Arte Contemporáneo del GEIIC, documento electrónico disponible en [http://ge-iic.com/index.php?option=com_content\&task=v iew\&id=732\&ltemid=63], consultado en julio del 2014 .

2014h Revista Ge-conservación, documento electrónico disponible en [http://ge-iic.com/ojs/index.php/revista], consultado en julio del 2014.

MECD

2014 "Premio Nacional de Conservación y Restauración", MECD (Ministerio de Educación, Cultura y Deportes), España, documento electrónico disponible en [http://www.mecd.gob. es/cultura-mecd/areas-cultura/patrimonio/premios/premiosrestauracion/premiados.html], consultado en julio del 2014.

Unirioja

2014 Dialnet, base de datos de la Universidad de La Rioja, documento electrónico [página web] disponible en [http:// dialnet.unirioja.es], consultado en julio del 2014. 


\section{Resumen}

La presente SECCIÓN ESPECIAL, concebida con motivo del quinto aniversario de Intervención, alienta una discusión crítica, ya necesaria, sobre el desarrollo y la actualidad de las publicaciones académicas periódicas en el sector patrimonial del mundo a partir de la presentación de dos iniciativas de vanguardia de origen ibérico: ECR, Estudos de Conservação e Restauro (Portugal) y Ge-conservación (España). Los análisis de sus editoras: Eduarda Silva Vieira y Rocío Bruquetas Galán, respectivamente, ofrecen una visión introspectiva de la génesis y consolidación de dos proyectos editoriales con identidades propias, hoy consolidados, en la que se subrayan los principales logros alcanzados y los retos que se vislumbran: doble contribución que, además de honrar los esfuerzos internacionales, concertantes, que se realizan en materia de difusión académica de la conservación-restauración en variadas lenguas -incluidos el castellano, el portugués y el inglés-, pone en su justo valor el impacto de la digitalización editorial que dota de mayor visibilidad a la literatura profesional y optimiza el intercambio científico y la colaboración interdisciplinaria a escala global.

En nuestro ámbito, sin duda, este escenario sin parangón conduce a la reflexión acerca de los nuevos paradigmas que transforman mundialmente la producción académica, el desarrollo científico, la investigación y la formación profesional, y nos llama a una nueva configuración de redes editoriales electrónicas para la región iberoamericana.

\section{Palabras clave}

difusión científica; publicaciones académicas periódicas; Estudos de Conservação e Restauro; ECR; Ce-conservación; patrimonio cultural; conservación-restauración

\section{Abstract}

The present SPECIAL SECTION, which was created to celebrate the fifth anniversary of Intervención, encourages a critical and necessary discussion about the development and actuality of academic periodical publications in the heritage worldwide. This contribution takes as a starting point the presentation of two forefront initiatives of Iberian origin: ECR, Estudos de Conservação e Restauro (Portugal) and Ge-conservación (Spain). The editors of the above mentioned journals —Eduarda Silva Vieira and Rocío Bruquetas Galán- provide in their respective analysis an inside view of the creation process and consolidation of two editorial projects with their own - now established - identity, while emphasizing their main achievements and looming challenges. Apart from honouring the international joint efforts that are done for the academic dissemination of conservation-restoration in various languages —including Spanish, Portuguese and English-, this double contribution puts into perspective the impact of digital publication that provides greater visibility of professional literature and optimizes scientific exchange, as well as interdisciplinary collaboration on global scale. This unparalleled scenario, undoubtedly, leads to reflection in our field on new paradigms that worldwide transform academic production, scientific development and vocational training and it calls for a new configuration of electronic editorial networks for the Ibero-American region.

\section{Key words}

scientific dissemination; academic periodical journals; Estudos de Conservação e Restauro; ECR; Ge-conservación; cultural heritage; conservation-restoration 